\title{
$\beta$-Glucans in food modify colonic microflora by inducing antimicrobial protein, calprotectin, in a Dectin-1-induced-IL-17F-dependent manner
}

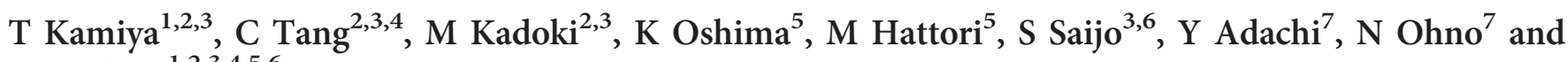
Y Iwakura ${ }^{1,2,3,4,5,6}$

Dectin-1 (gene symbol: Clec7a) is a receptor for $\beta$-glucans that play an important role for the host defense against fungi. Recently, we showed that $\mathrm{Clec7a}^{-1-}$ mice are resistant against dextran sodium sulfate (DSS)-induced colitis because of regulatory T-cell population expansion in the colon. The regulatory T-cell expansion is caused by expansion of commensal Lactobacillus murinus whose growth is suppressed by an antimicrobial protein, calprotectin S100A8/A9. In this report, we showed that S100A8 was mainly produced by mouse colonic epithelial cells. S100A8 was not induced directly by Dectin-1 but by Dectin-1-induced cytokines, especially interleukin-17F (IL-17F), that were produced by several types of innate immune cells including $\mathrm{CD}_{11 \mathrm{c}^{+}} / \mathrm{CD} 1 \mathrm{~b}^{+}$myeloid cells in colonic lamina propria. S100A8/A9 heterodimer preferentially suppressed the growth of $L$. murinus that was increased in both $C l e c 7 a^{-\prime-}$ and $I 117 f^{-\prime-}$ mice. Furthermore, similar expansion of $L$. murinus and DSS-colitis resistance were observed in mice fed with $\beta$-glucanfree food. These observations suggest that food-derived $\beta$-glucans control the specific commensal microbiota via the Dectin-1-IL-17F-calprotectin axis to maintain the intestinal homeostasis.

\section{INTRODUCTION}

Our intestinal tract is colonized by many types of microbiota. The number and diversity of these microbes are always changing in any given moment throughout our life, and the majority of these organisms are not harmful invaders but cohabitants living with us as their one-and-only home. ${ }^{1-3}$ Indeed, our metabolic and immune functions are largely dependent on our intestinal microflora. By activating host antimicrobial innate immune mechanisms $\mathrm{s}^{4,5}$ and by directing the development of adequate adaptive immune cells of the mucosal and systemic immune system, commensal microbes contribute to the host defense against pathogens ${ }^{6}$ and host immune homeostasis. ${ }^{7,8}$

Recent studies have revealed the importance of pattern recognition receptors (PRRs), which recognized pathogenassociated molecular patterns, in the host defense against pathogens and intestinal immune homeostasis. ${ }^{9,10}$ C-type lectin receptors (CLRs) is a group of such PRRs and bind carbohydrate structures on pathogens, such as fungi and mycobacteria, in a $\mathrm{Ca} 2{ }^{+}$-dependent manner, ${ }^{11}$ providing an important mechanism for the protection against these pathogens. ${ }^{12-15}$ Dectin-1 (gene symbol: Clec7a) is one of type II CLRs that contains a carbohydrate recognition domain in its extracellular carboxyl terminus and an immunoreceptor tyrosine-based activation motif in its intracellular amino terminus, ${ }^{16}$ and highly expressed in dendritic cells, macrophages (M $\phi s)$, and neutrophils. Previously, the group of Brown ${ }^{17}$ and we showed that Dectin- 1 is the receptor for $\beta-1,3$ linked glucans with $\beta-1,6$-linked branches ( $\beta$-glucans) and plays an important role for the host defense against fungi by producing cytokines and reactive oxygen species. ${ }^{18}$ We have also shown that Dectin-2 (gene symbol: Clec4n) is the receptor

${ }^{1}$ Graduate School of Science, University of Tokyo, Tokyo, Japan. ${ }^{2}$ Research Institute for Biomedical Sciences, Tokyo University of Science, Chiba, Japan. ${ }^{3}$ Center for Experimental Medicine and Systems Biology, The Institute of Medical Science, University of Tokyo, Tokyo, Japan. ${ }^{4}$ Core Research for Evolutional Science and Technology (CREST), Japan Science and Technology Agency (JST), Saitama, Japan. ${ }^{5}$ Graduate School of Frontier Sciences, University of Tokyo, Chiba, Japan. ${ }^{6}$ Department of Molecular Immunology, Chiba University, Chiba, Japan and ${ }^{7}$ School of Pharmacy, Tokyo University of Pharmacy and Life Science, Tokyo, Japan. Correspondence: Y Iwakura (iwakura@rs.tus.ac.jp) 
for $\alpha$-mannans and important for the host defense against fungal infection. ${ }^{14}$ Although Dectin-1 and Dectin-2 are expressed in the colonic lamina propria (cLP) and Peyer's patches in the small intestine and $\beta$-glucans and $\alpha$-mannans are contained in many foods such as mushrooms, yeasts, and seaweeds, the role of these CLRs in the intestinal immune system have not been elucidated completely.

Antimicrobial proteins/peptides (AMPs) are a group of proteins that have broad antimicrobial activity against bacteria and other microorganisms, being secreted by intestinal epithelial cells, Paneth cells, and innate immune cells. ${ }^{19,20}$ AMPs are considered to be a phylogenetically ancient form of innate immunity and provide host defense at the skin and mucosal surfaces ${ }^{19}$ by aggregating pathogens or permeabilizing pathogen membrane. ${ }^{21}$ AMPs also play an important role in regulating intestinal commensal microflora. Upon infection, signaling from PRRs on mucosal immune cells, such as dendritic cells or $\mathrm{M} \phi \mathrm{s}$, induces local production of cytokines. Then, cytokines activates production of various AMPs, ${ }^{22}$ such as cationic proteins (defensin family), peptidoglycan binding proteins (REG proteins), and others. ${ }^{20,23,24}$ Cytokine-mediated regulation of AMP production has been well demonstrated in recent studies, ${ }^{20}$ and interleukin 17 (IL-17) family cytokines produced by Thelper type $17, \gamma \delta \mathrm{T}$, and innate immune cells are important inducers for skin and mucosal AMPs. ${ }^{23,24}$ Under healthy conditions, basal levels of AMPs are important for the maintenance of intestinal barrier function and commensal composition, although the precise molecular mechanisms of how AMP secretion is controlled under physiological conditions remain obscure.

Recently, we reported that Dectin-1 deficiency causes an increase of a specific lactobacillus species (Lactobacillus murinus) in the colon because of a decrease of a group of AMPs, and suppresses the development of colitis by promoting regulatory T-cell expansion in a lactobacillus-dependent manner. ${ }^{25}$ However, the mechanism of how AMPs are induced by Dectin- 1 signaling and the source of $\beta$-glucans that activate Dectin-1 in the intestine remain largely obscure. In this study, we analyzed the detailed mechanisms by which Dectin-1 signaling controls intestinal microbiota and identified the source of $\beta$-glucans in the intestine.

\section{RESULTS}

\section{L. murinus is increased in colonic microflora of $\mathrm{Clec7a}^{-1-}$ mice}

To investigate the roles of CLRs in regulating microflora composition, we analyzed the colonic microflora populations in feces by bacterial 16S ribosomal DNA (rDNA) sequence analysis in wild-type (WT), Dectin-1 deficient $\left(\mathrm{Clec} \mathrm{a}^{-1-}\right)$, Dectin-2 deficient (Clec4n ${ }^{-1-}$ ), and mice deficient for MyD88 $\left(M y d 88^{-1-}\right)$, an adaptor molecule for Toll-like receptors (TLRs). The proportion of Lactobacillaceae family was 5 times higher in fecal microflora of $\mathrm{Clec}_{\mathrm{Ha}} \mathrm{a}^{-1-}$ mice than that of WT mice (Figure 1a and Supplementary Figure S1a). Changes of colonic microflora composition of mice deficient in $M y d 88^{-/-}$ were less prominent under our experimental conditions, although several TLRs are known to recognize commensal bacteria and transmit signals through MyD88. We found that the proportion of L. murinus NBRC14221 in total Lactobacillaceae was dramatically increased in $\mathrm{Clec}_{7} \mathrm{a}^{-1-}$ and this strain was also significantly increased in $\mathrm{Clec}_{4} \mathrm{n}^{-/-}$and

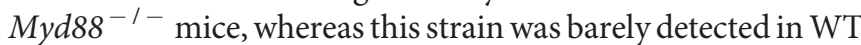
mice (Figure 1b and Supplementary Figure S1b). Proportion of Lactobacillus johnsonii GAL-2, another strain of the lactobacillus family, was also increased in $\mathrm{Clec}_{7 a^{-1-}}$, Clec $4 n^{-1-}$, and Myd88 $8^{-1-}$ mice. We showed recently that $L$. murinus can induce regulatory T cells in the intestine, but $L$. johnsonii has not such inducing activity. ${ }^{25}$

When we co-fostered $C l e c 7 a^{-1-}$ mice with WT mice, no increase of L. murinus was observed in $C l e c 7 a^{-1-}$ mice (Supplementary Figure S2a), but when we separately housed these mice after weaning, L. murinus content in $C l e c 7 a^{-1-}$ mice gradually expanded compared with that in WT mice (Supplementary Figure S2b). These results indicate that Dectin-1 regulates the colonization of L. murinus.

\section{Production of AMP S100A8, which specifically suppresses L. murinus, is reduced in $\mathrm{Clec}^{-1-}$ colonic epithelial cells (cECs)}

Then, we investigated the reason why L. murinus proportion is increased in these Dectin-1-deficient mice. Because AMPs hold potent antimicrobial activities, we first analyzed AMP gene expression in the colon of WT and Clec $7 a^{-1-}$ mice. We found that the expression levels of calprotectin S100A8 (S100a8), but not S100A9 (S100a9) or other AMPs such as RegIII family members (Reg3b, Reg3g), Lipocalin-2 (Lcn2), $\alpha$-defensins- 1 and -4 (Defa 1,4$), \beta$-defensin-1 and -4 (Defb1, 4), and lysozymes ( $L y z 1, L y z 2)$, were significantly lower in the cEC layer of Clec $7 a^{-1-}$ mice than that of WT mice (Figure 2a).

To identify the sources of S100A8, we analyzed S100A8 protein expression by flow cytometry, and found that S100A8 was mainly expressed in CD $45^{-}$EpCAM- $1^{+}$cECs (Figure 2b and Supplementary Figure S3), consistent with previous report that S100A8 is produced by myeloid cells and epithelial cells. ${ }^{26-28}$ This S100A8 ${ }^{+}$cEC population was decreased in $\mathrm{Clec} 7 \mathrm{a}^{-1-}$ mice compared with WT mice (Figure 2b). S100a8 expression in cLP was also decreased (Figure 2c), although these data are not statistically significant because of the scarcity of the number of S100A8-expressing cells in cLP layer compared with cEC layer (Figure 2d). These results indicate that Dectin-1-induced S100A8 is mainly produced by cECs.

S100A8/A9 heterodimer, but not S100A8 monomer, is reported to suppress the growth of Gram-positive bacteria such as Staphylococcus spp. ${ }^{29}$ We found that S100A8 + A9, but not S100A8 only, significantly suppressed the growth of L. murinus in vitro (Figure 2e), whereas lipocalin-2 had no L. murinussuppressing activity (Figure 2f). We also found that this S100A8 + A9 could not suppress the growth of Escherichia coli, Lactococcus lactis, or even L. johnsonii (Figure 2g), suggesting that calprotectin preferentially controls the L. murinus population. 
a
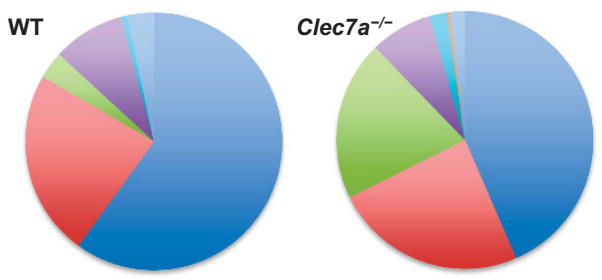

b wT

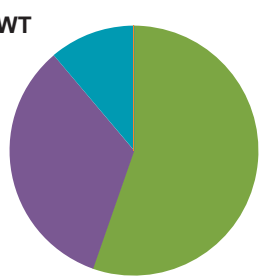

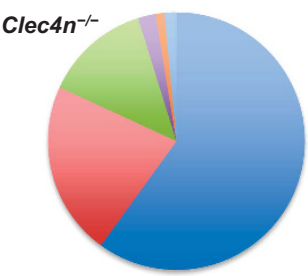

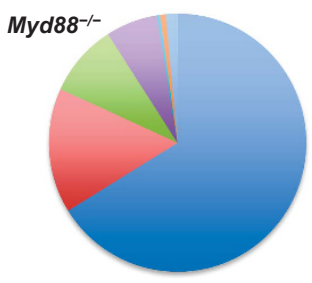

- Lachnospiraceae

- Ruminococcaceae

- Lactobacillaceae

Porphyromonadaceae

- Clostridiaceae

Desulfovibrionaceae

- Others

Figure 1 The proportion of Lactobacillus murinus in colonic microbiota is increased in Clec7a ${ }^{-1}$ mice. (a) Fecal microfloras from wild-type (WT), Clec $7 a^{-1-}$, Clec $4 n^{-1-}$, and Myd88 ${ }^{-1-}$ mice were analyzed by the $16 \mathrm{~S}$ ribosomal DNA (rDNA) sequence method, and proportions of major commensal bacteria are presented ( $n=3 /$ group). (b) Frequencies of major Lactobacillus strains in total Lactobacillaceae from WT, Clec7a ${ }^{-/-}, C l e c 4 n^{-1-}$, and

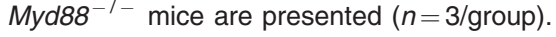
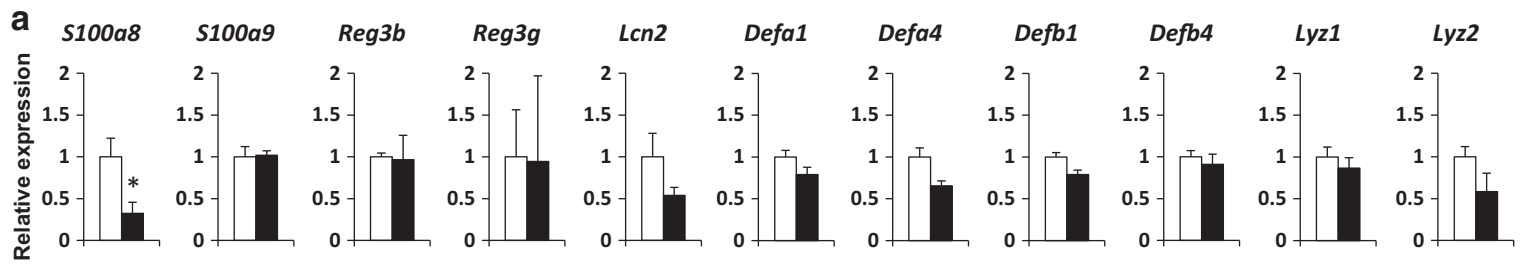

b
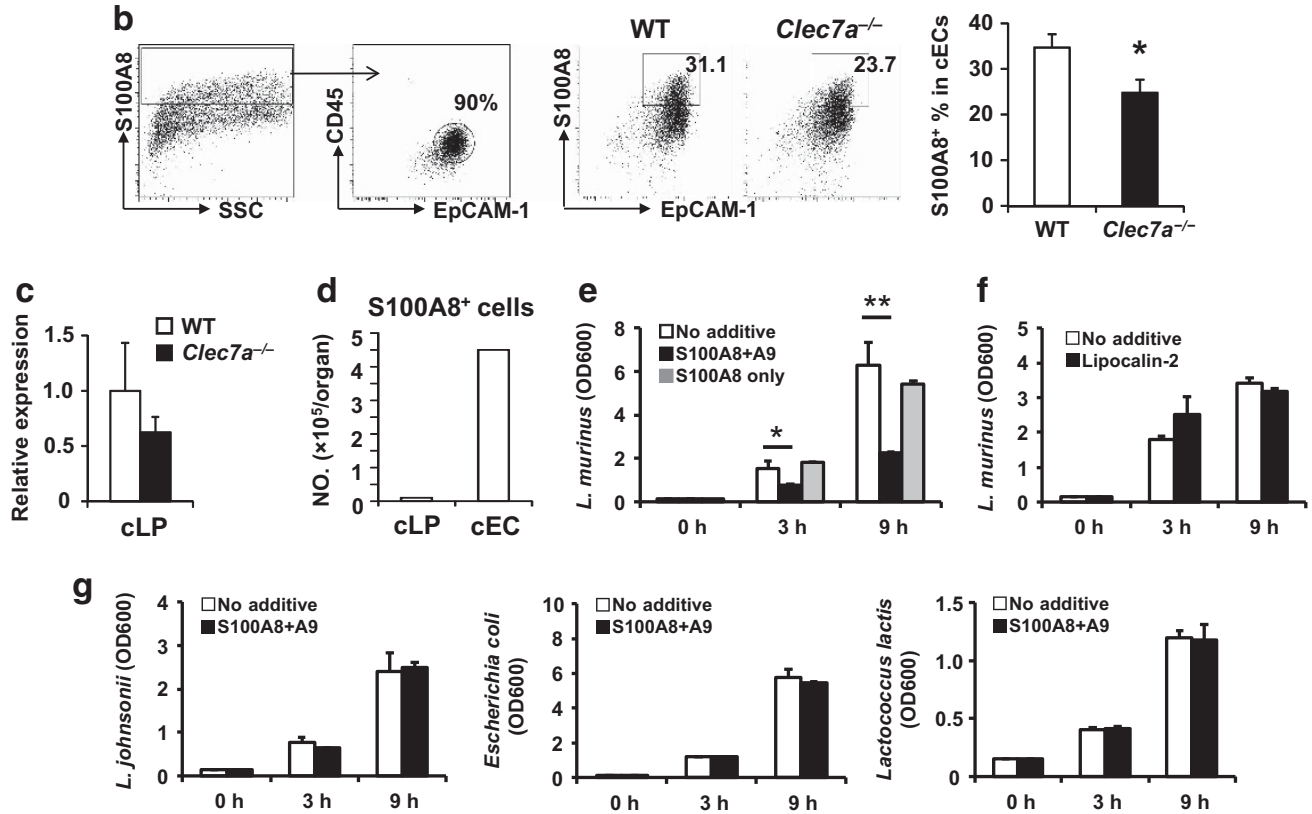

Figure 2 Production of calprotectin S100A8, which preferentially suppresses the growth of Lactobacillus murinus, is impaired in colonic epithelial cells (cECs) of Clec7a ${ }^{-1-}$ mice. (a) Expression of antimicrobial protein/peptide (AMP) genes (S100A8 and 9 (S100a8 and S100a9), Reglll $\beta$ and $\gamma$ (Reg3b and Reg3g), Lipocalin-2 (Lcn2), $\alpha$ - and $\beta$-defensins (Defa1, Defa4, Defb1, and Defb4), and Lysozyme-1 and 2 ( $L y z 1$ and $L y z 2)$ ) in cEC layer of wild-type (WT) and $\mathrm{Clec7a}^{-1}$ mice were measured by quantitative real-time reverse transcription PCR (qRT-PCR; $n=3-4 / \mathrm{group}$ ). (b) Cells from cEC layer of WT or $\mathrm{Clec7a}^{-1-}$ mice were analyzed for S100A8 production by flow cytometry ( $n=3 /$ group). Data are representatives of two independent experiments. (c) S100a8 mRNA expression in colonic lamina propria (cLP) layer of WT or Clec7a ${ }^{-1-}$ mice was examined by qRT-PCR. (d) Number of S100A8 ${ }^{+}$cells in WT CLP or cEC layer was measured by flow cytometry. (e-g) L. murinus, L. johnsonii, Escherichia coli, or Lactococcus lactis were cultured in the presence of recombinant $\mathrm{S} 100 \mathrm{~A} 8\left(5 \mu \mathrm{g} \mathrm{ml}^{-1}\right)$ or $\mathrm{S} 100 \mathrm{~A} 8+\mathrm{A} 9\left(1: 1\right.$ mixture, $5 \mu \mathrm{g} \mathrm{ml}^{-1}$ of each peptide) $(\mathbf{e}, \mathbf{g})$, or lipocalin-2 $\left(5 \mu \mathrm{g} \mathrm{ml} \mathrm{m}^{-1}\right)(\mathbf{f})$, and bacterial growth was measured at 3 and $9 \mathrm{~h}$ of cultivation with a spectrophotometer. Data in $\mathbf{a}$ are representatives of three, and in $\mathbf{b}-\mathbf{g}$ are representatives of two independent experiments. Data in $\mathbf{a}-\mathbf{g}$ are expressed as mean \pm s.d. ${ }^{\star} P<0.05,{ }^{\star \star} P<0.01$ vs. control. 


\section{Dectin-1 signaling induces proinflammatory cytokines in CLP}

Lipopolysaccharide, a TLR4 ligand, induces S100a8 expression in macrophages, ${ }^{30}$ and cytokines such as interferon- $\gamma$, tumor necrosis factor- $\alpha$ (TNF- $\alpha)$, or IL-17C can also induce S100A8. ${ }^{31,32}$ As Dectin-1 signaling also induces various cytokine production, ${ }^{18,33}$ we examined the possibility that colonic S100A8 expression is mediated by Dectin-1-inudced cytokines. Using quantitative real-time reverse transcription PCR (qRT-PCR), we found that the expression of Ill7f, but not of Tnfa, Il12p35, Ilo, or other IL-17 family members (Ill7a, $I l 17 b, I l 17 c, I l 17 d)$, was significantly lower in $\mathrm{CLP}$ of Clec $7 a^{-1-}$ mice than that of WT mice (Figure 3a). Expression of Il22 also tended to be lower in $C l e c 7 a^{-1-}$ colon (Figure 3a). Furthermore, we showed that IL-17F was mainly produced by $\mathrm{CD}_{4} 5^{+}$leukocytes in $\mathrm{CLP}$, and these $\mathrm{IL}-17 \mathrm{~F}^{+} \mathrm{CD} 45^{+}$ leukocytes were significantly decreased in $\mathrm{Clec} \mathrm{a}^{-1-}$ mice (Figure 3b,c). S100A8 is mainly secreted by cECs (Figure 2b), but not $\mathrm{CD} 45^{+}$leukocytes that produce IL-17F (Figure 3d), suggesting that S100A8 is induced by IL-17F in a paracrine manner. On the other hand, S100A9 is constitutively secreted by cECs (Supplementary Figure S4).

\section{S100A8 production in cECs is induced by CD11c ${ }^{+} / C D 11 b^{+}$ cell-derived IL-17F}

Dectin-1 was highly expressed in $\mathrm{CD}_{1} 1 \mathrm{~b}^{+} \mathrm{CD} 11 \mathrm{c}^{-}$and

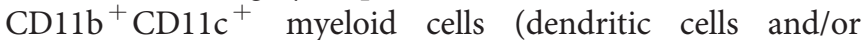
$\mathrm{M} \phi s$ ) in cLP (Figure 4a), but not in cECs. ${ }^{27}$ Thus, we hypothesized that IL-17F induced by Dectin-1 signaling in these myeloid cells indirectly mediated the induction of S100A8 in cECs. To confirm this, we purified cLP CD11c ${ }^{+} \& \mathrm{CD} 11 \mathrm{~b}^{+}$ cells from WT and Clec $7 a^{-1-}$ mice, cultured them for $6 \mathrm{~h}$ with curdlan, a particle form of $\beta$-glucans, and then examined the AMP-inducing activity of the culture supernatants in cECs. Interestingly, S100a8 expression was specifically induced in cECs by the conditioned medium, but the induction was not observed in S100a9, Defb1, or other AMPs (Figure 4b and Supplementary Figure S5a). Furthermore, the $\$ 100 a 8$ induction was significantly reduced in $\mathrm{Clec} \mathrm{a}^{-/-}$cLP cellderived conditioned medium (Figure 4c). These results suggest the presence of some factors in the conditioned medium that can preferentially induce S100a8.

Then, we investigated cytokine production in curdlantreated cLP cells. As shown in Figure 4d, the expressions of Il17f and Tnfa, but not Il22 or Il23p19, in cLP CD11c ${ }^{+} \& \mathrm{CD} 11 \mathrm{~b}^{+}$ cells were significantly higher in WT cLP cells than in $C l e c 7 a^{-/-}$ cLP cells. We then stimulated colon organ pieces from WT mice with recombinant mouse IL-10, Il-17F, IL-22, IL-23, or TNF- $\alpha$, and found that $S 100 a 8$ expression was upregulated by IL-17F, IL-22, and IL-23 in a dose-dependent manner (Figure 4e,f). Furthermore, we found that $\$ 100 a 8$ expression was only induced by recombinant IL-17F and IL-22 in the primary culture of colonic epithelium (Figure $4 \mathrm{~g}$ and Supplementary Figure S5b). Because only IL-17F, but not IL-22, was significantly reduced in $C l e c 7 a^{-1-}$ colon (Figure 3a), these results confirm that IL-17F produced by cLP $\mathrm{CD}_{11 c^{+}} \& \mathrm{CD} 11 \mathrm{~b}^{+}$cells is mainly involved in the induction of S100A8 in mouse cECs. We further found that addition of rIL-17F restored the S100A8 expression in the colon of $\mathrm{Clec} \mathrm{a}^{-1-}$ mice (Supplementary Figure S5e), supporting the notion that Dectin-1-induced IL-17F is involved in this calprotectin induction.
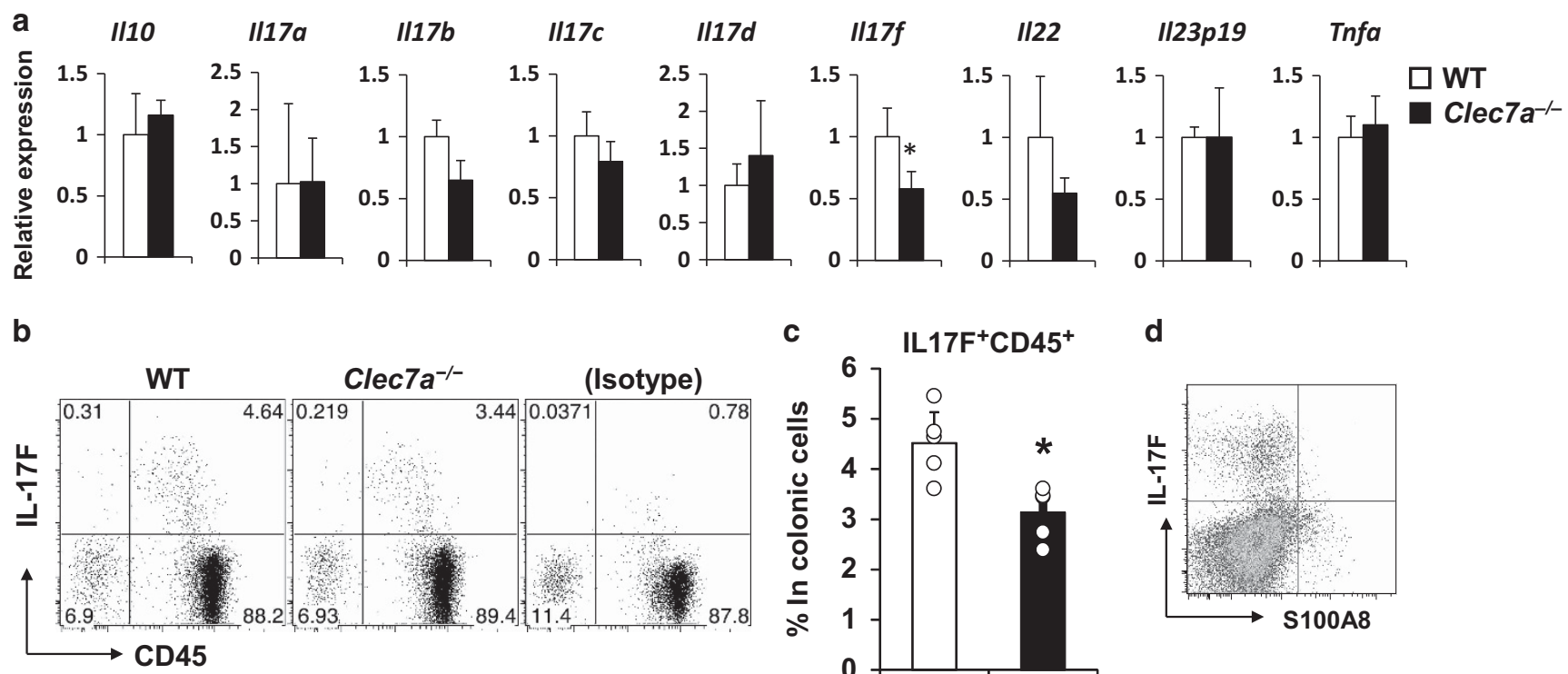

C

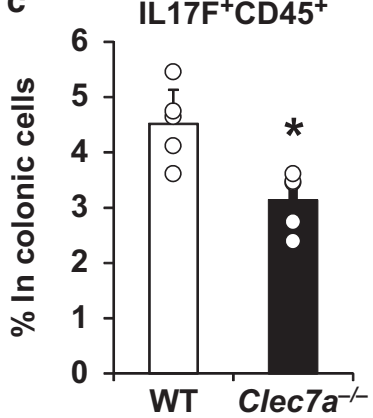

d

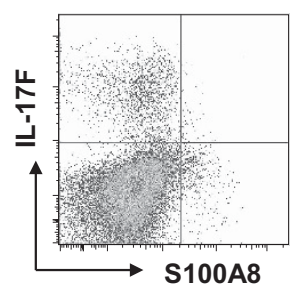

Figure 3 Interleukin-17F (IL-17F) production is decreased in colonic lamina propria (cLP) of Clec7a ${ }^{-/-}$mice. (a) Cytokine gene expression in cLP was examined by quantitative real-time reverse transcription PCR (qRT-PCR), and expression levels in $\mathrm{Clec7a}^{-1-}$ mice are indicated relative to those in wild-type (WT) mice ( $n=3-8 /$ group). (b,c) IL- $17 \mathrm{~F}^{+} \mathrm{CD} 45^{+}$cell population in cLP layer from WT or Clec7a ${ }^{-1-}$ mice was examined by flow cytometry. ( $n=4-5 /$ group). (d) $\mathrm{S} 100 \mathrm{~A}^{+}$and IL-17F ${ }^{+}$cells in whole colonic cells from WT mice were examined by flow cytometry. Data are representatives of two independent experiments, and in $\mathbf{a}$ and $\mathbf{c}$ are expressed as mean \pm s.d. ${ }^{*} P<0.05$ vs. WT control. 
a

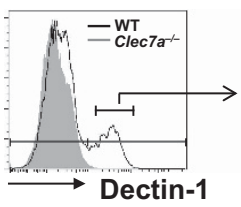

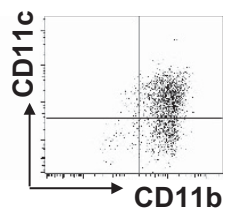

C

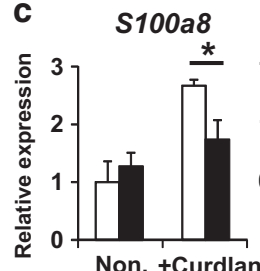

S100a9

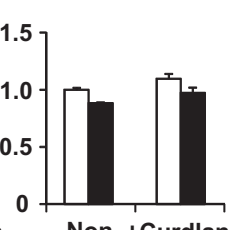

Defb1

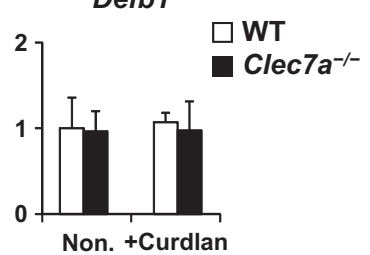

Non. +Curdlan
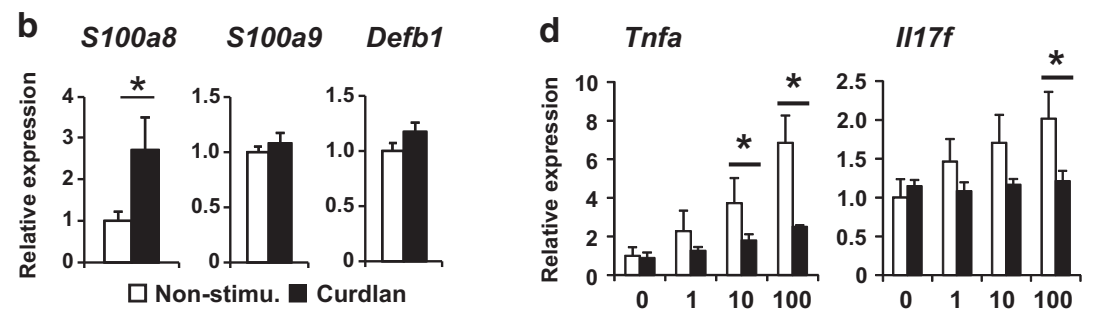

* $1123 p 19$
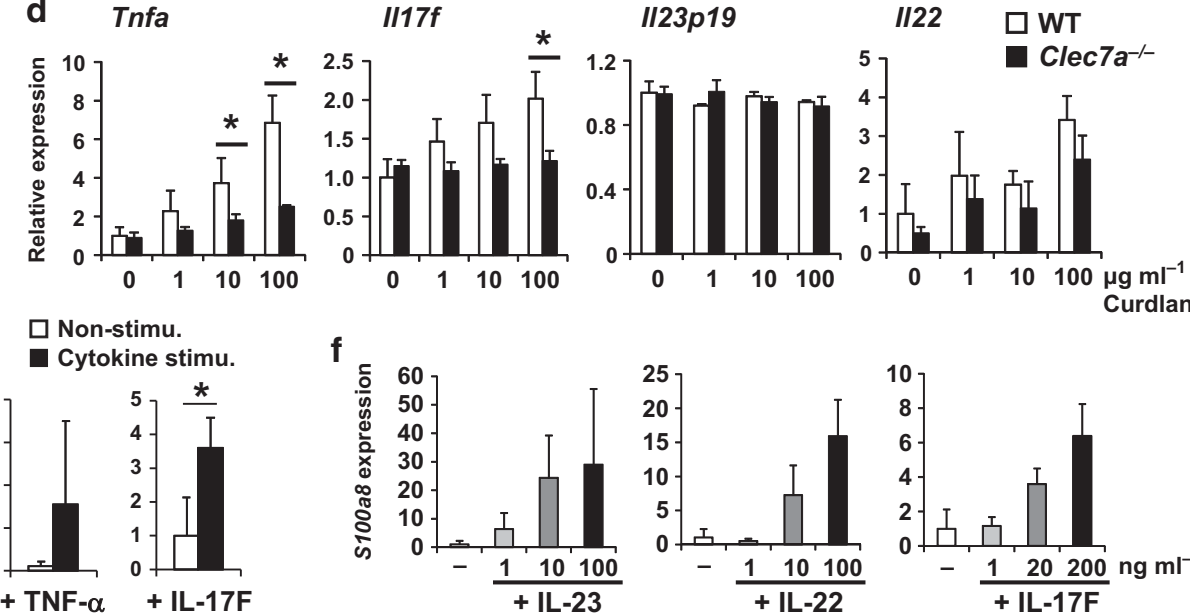

f
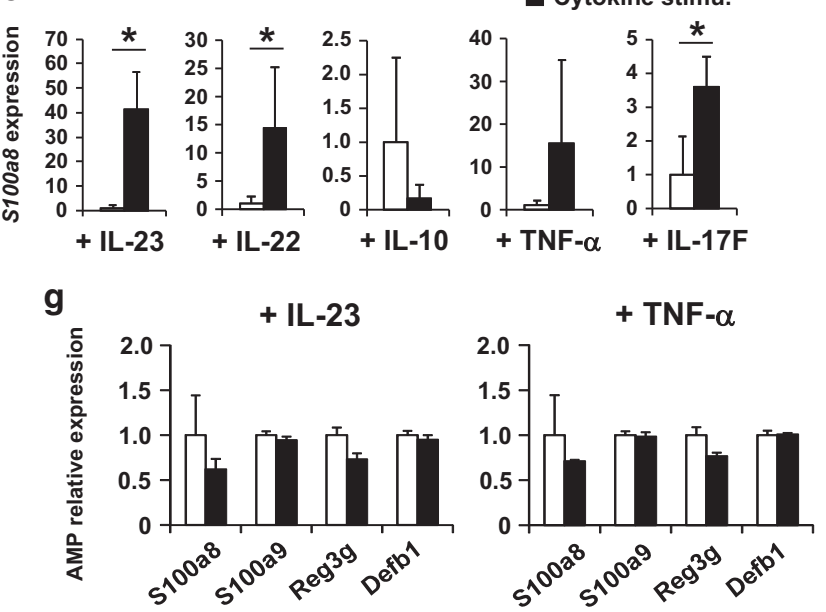
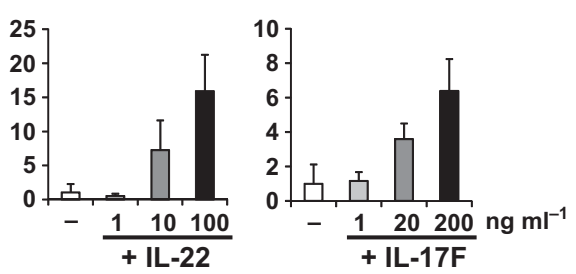

+ IL-17F
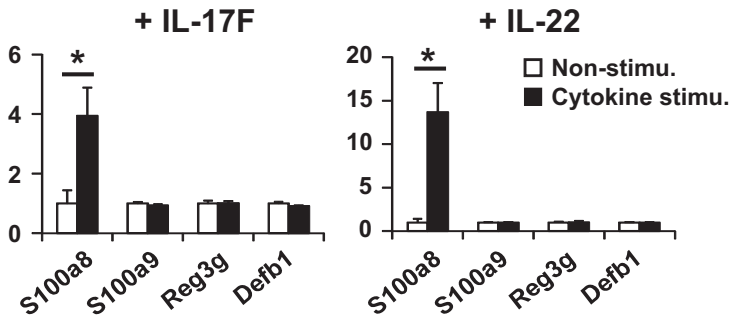

Figure 4 Dectin-1 signaling promotes S100A8 production through induction of interleukin-17F (IL-17F) in colonic lamina propria (cLP) CD11c ${ }^{+}$\&CD11b myeloid cells. (a) CD11b and CD11c expression on Dectin-1 $1^{+}$CLP cells from wild-type (WT) or Clec7a ${ }^{-1-}$ mice were analyzed by flow cytometry. (b,c) Colonic LP-derived CD11 ${ }^{+}$and CD11c ${ }^{+}$cells purified by magnetic-activated cell sorting (MACS) from (b) WT or (c) WT and Clec7a ${ }^{-1-}$ mice were stimulated with curdlan for $72 \mathrm{~h}$, and culture supernatants were then used to stimulate colonic epithelial cells (cECs) for $6 \mathrm{~h}$. After the stimulation, indicated antimicrobial protein/peptide (AMP) gene expression was determined by quantitative real-time reverse transcription PCR (qRT-PCR). (d) CD11b ${ }^{+}$and

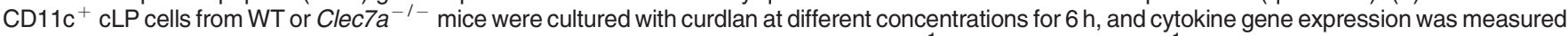
by qRT-PCR. (e,f) Pieces of WT colon were stimulated with indicated cytokines $\left(20 \mathrm{ng} \mathrm{ml}^{-1}\right.$ in e and $1-100 \mathrm{ng} \mathrm{ml}^{-1}$ in f) for (e) $6 \mathrm{~h}$ or (f) $12 \mathrm{~h}$, and $S 100 \mathrm{a} 8$ expression was determined by qRT-PCR. (g) WT cECs were incubated with indicated cytokines $\left(100 \mathrm{ng} \mathrm{ml}^{-1}\right)$ for $6 \mathrm{~h}$, and S100a8, S100a9, Reg3g, and Defb1 gene expression was determined by qRT-PCR ( $n=3 /$ group). Data in $\mathbf{b}-\mathbf{g}$ are representatives of two independent experiments and are expressed as mean \pm s.d. ${ }^{*} P<0.05$ vs. control.

\section{S100A8 expression is reduced in $I 117 f^{-I-}$ mouse colon as in $\mathrm{Clec7a}^{-1-}$ colon}

Then, we examined S100A8 expression in $I l 17 f^{-1-}$ mouse colon to show that IL-17F is responsible for the induction of S100A8. S100A8-producing cECs were significantly, although not completely, decreased in $I l 17 f^{-1-}$ mice compared with that in WT mice (Figure 5a,b). The $\$ 100 a 8$ expression level in cECs was significantly decreased in $I l 17 f^{-1-}$ mice, but not in $I l 17 a^{-/-}$mice (Figure 5c and Supplementary Figure S5c,d), suggesting that IL-17A is not required for S100A8 induction in this setting. S100A 8 production from $\mathrm{cECs}$ was induced by the culture supernatant of curdlan-stimulated CD $11 c^{+} \& C D 11 b^{+}$ cells from CLP, and this induction was cancelled by the deficiency of IL-17F (Figure 5d). These results further support the idea that S100A8 production in cECs is mainly induced by IL-17F. Consistent with these observations, $16 \mathrm{~S}$ rDNA levels of L. murinus were significantly increased in the $I l 17 f^{-1-}$ mouse colon (Figure 5e), similar to Clec7a $a^{-1-}$ mice (Figure 1b).

\section{Commensal microbiota do not express Dectin-1 ligands}

We then investigated the source of Dectin-1 ligands in mouse intestine. It was reported that mouse colon is inhabited by commensal fungi such as Candida tropicalis that express $\beta$ glucans as a cell wall component. ${ }^{2}$ To search for Dectin-1 ligands in mouse intestine, colonic microbiota from WT mouse feces were incubated with $\beta$-glucan-recognizing protein derived from Plodia interpunctella $(\mathrm{Pi}-\mathrm{BGRP})^{34}$ and analyzed by flow cytometry. Although Pi-BGRP binding resulted in a 

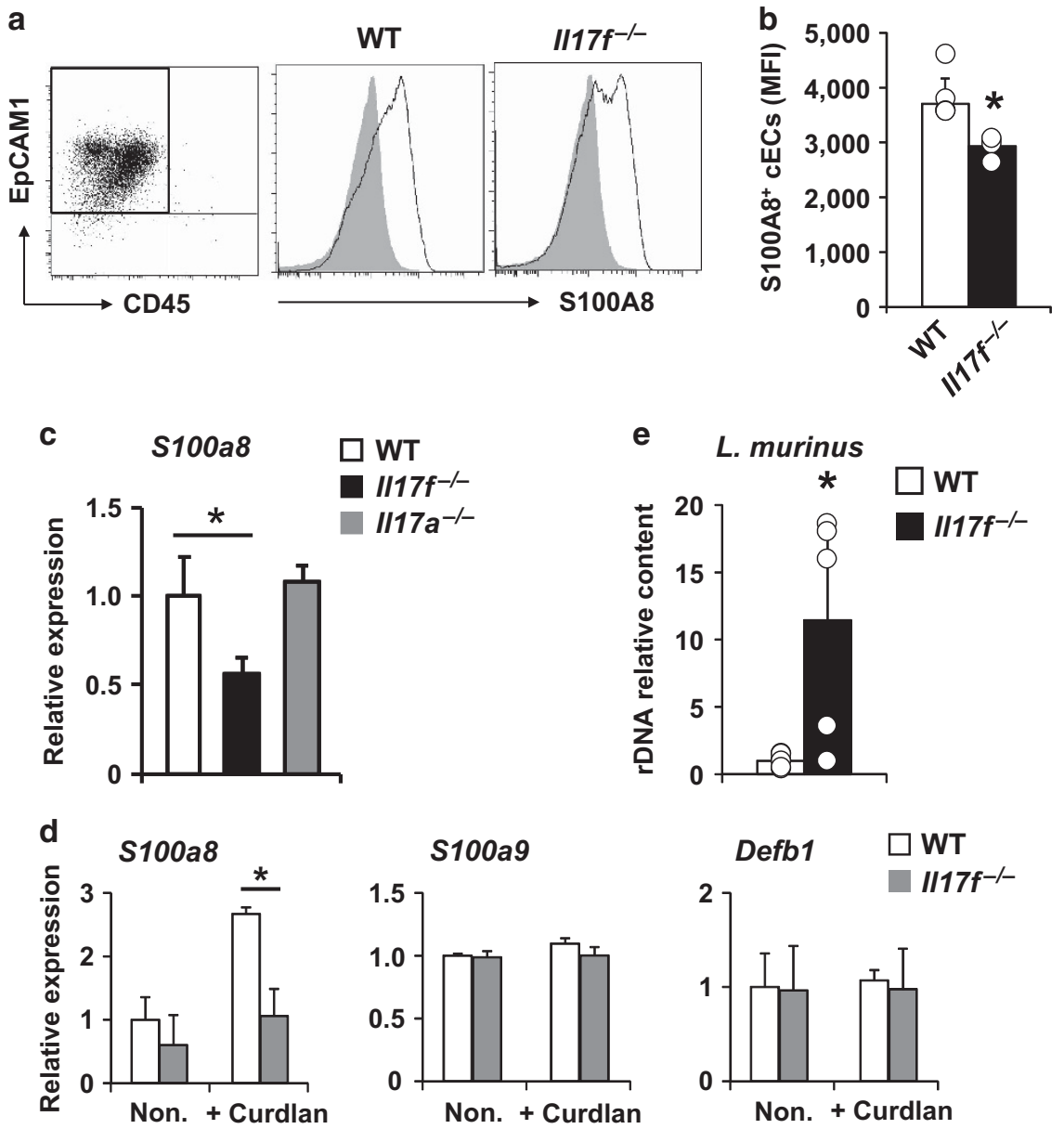

Figure 5 S100A8 production in the colon is reduced in $I / 17 f^{-1-}$ mice. (a,b) S100A8 expression on CD45-EpCAM-1 ${ }^{+}$colonic epithelial cells (cECs) of wild-type (WT) or $I / 17 f^{-1-}$ mice was examined by flow cytometry (filled histogram: isotype control; solid line: anti-S100A8) (a), and the mean fluorescence intensity (MFI) is shown in (b) $\left(n=4-5 /\right.$ group). (c) S100a8 expression in cEC from WT, $/ 17 f^{-l-}$ mice, or $/ 117 a^{-1-}$ mice $(n=3 /$ group) was determined by quantitative real-time reverse transcription PCR (qRT-PCR). (d) Colonic lamina propria (LP) CD11b ${ }^{+}$and CD11c ${ }^{+}$cells from WT or $1 / 17 f^{-1-}$ were stimulated with curdlan for $72 \mathrm{~h}$, and the culture supernatants were used to stimulate WT cECs for $6 \mathrm{~h}$. Then, indicated antimicrobial protein/ peptide (AMP) gene expression in cECs was determined by qRT-PCR. (e) Content of Lactobacillus murinus $16 \mathrm{~S}$ ribosomal DNA (rDNA) in feces from WT or $1 / 17 f^{-1-}$ mice was determined by qRT-PCR, and the relative contents in $I / 17 f^{-1-}$ mice to that in WT mice are shown $(n=4-5 / g r o u p)$. Data in $b-e$ are representatives of two independent experiments and are expressed as mean \pm s.d. ${ }^{*} P<0.05$ vs. WT control.

little intensity shift when binding was examined without blocking of nonspecific binding, this shift was cancelled after pretreatment with nonimmune goat serum (Figure 6a). Because Candida albicans was efficiently recognized by PiBGRP (Figure 6a), these results suggest that no commensal fungi or other Dectin-1 ligand-expressing microorganisms are present in our mouse intestine.

Alcaligenes faecalis var. myxogenes produces curdlan, ${ }^{35}$ and is used in industry to produce this substance in large amount. ${ }^{36}$ However, it was not known whether or not $A$. faecalis subsp. faecalis isolated from mouse intestine can produce curdlan. Thus, we examined this by the TNF-inducing activity of $A$. faecalis upon stimulation of WT or Clec7a ${ }^{-1-}$ M $\phi$ s. As shown in Figure 6b. TNF production was impaired in $M y d 88^{-1-}$ $\mathrm{M} \phi \mathrm{s}$, but not $\mathrm{Clec} 7 a^{-1-} \mathrm{M} \phi \mathrm{s}$, after A. faecalis stimulation, suggesting that TNF production is induced by TLR signaling, but not by Dectin-1 signaling. Furthermore, S100A ${ }^{+}$cEC population in A. faecalis-monocolonized mice was similar to that in germ-free or specific-pathogen-free mice (Figure 6c), indicating that $A$. faecalis or other commensal microbiota do not express Dectin-1 ligands.

\section{Food is the main source of $\beta$-glucans}

Mouse food commonly contains yeast extract as a nutrition. We then examined the possibility that food-derived $\beta$-glucans activate Dectin- 1 in mouse intestine. We analyzed colonic AMP expression in mice fed with $\beta$-glucan-free diet (BGFD) or normal diet (ND), and found that $\$ 100 a 8$ expression was significantly decreased in cECs of BGFD-fed mice compared with that of ND-mice (Figure 7a). Il17f expression was downregulated in CLP after BGFD administration (Supplementary Figure S6). Furthermore, L. murinus rDNA content was increased in BGFD-fed mice (Figure 7b), similar to $C l e c 7 a^{-1-}$ or $I l 17 f^{-1-}$ mice. These results suggest that $\beta$-glucans from food control L. murinus population by inducing S100A8. 
a

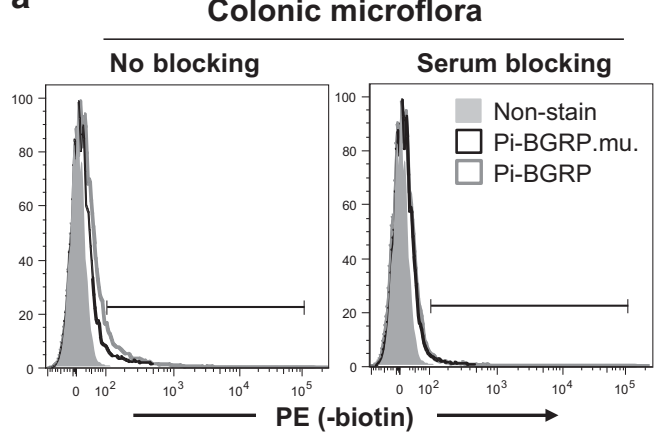

b

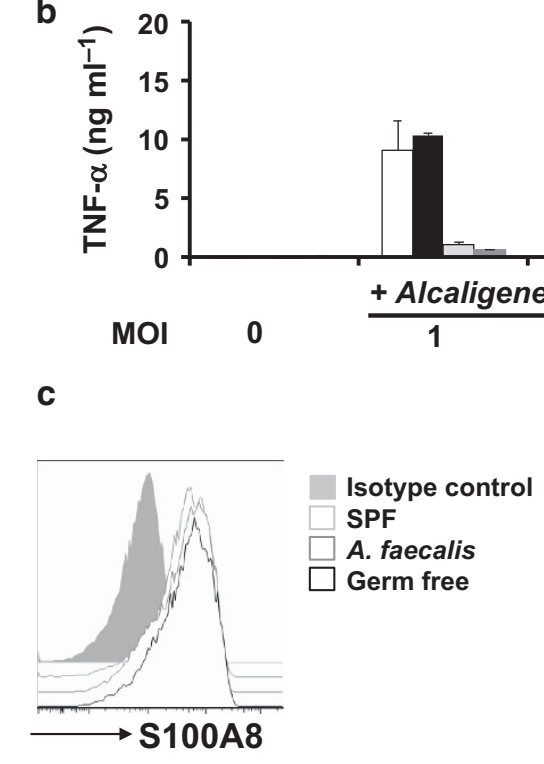

Candida albicans

(serum blocking)

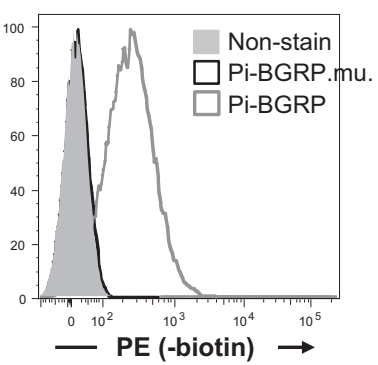

$\square$ WT

Clec7a ${ }^{-/-}$

MyD88

$\mathrm{Clec7a}^{-/-} \mathrm{MyD88^{-/- }}$

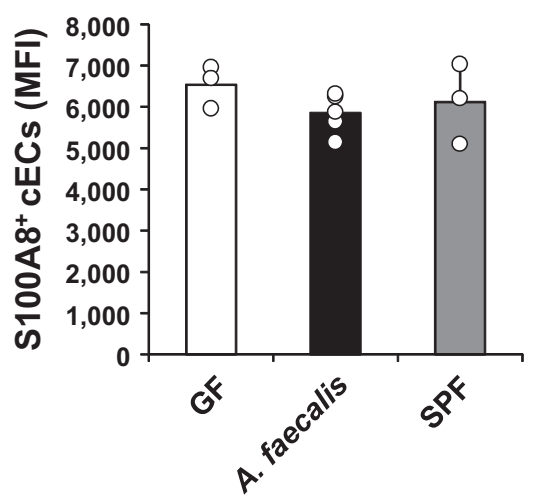

Figure 6 Dectin-1 ligands are not detected in intestinal commensal microbiota. (a) Wild-type (WT) mouse fecal microbiota or Candida albicans (SC5314) were pretreated with non-immune goat or rabbit serum to block nonspecific binding. Then, they were treated with PE-labeled $\beta$-glucanrecognizing protein (Pi-BGRP) or the mutant form of the $\beta$-glucan recognition site of Pi-BGRP (Pi-BGRP.M), and PE ${ }^{+}$population was determined by flow

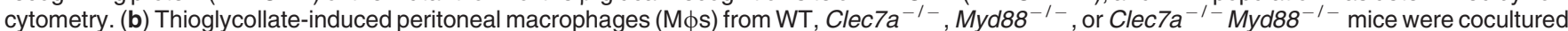
with live Alcaligenes faecalis (multiplicity of infection (MOI): 1 or 10 ) for $24 \mathrm{~h}$ and the tumor necrosis factor- $\alpha$ (TNF- $\alpha$ ) concentration in the culture supernatant was determined by enzyme-linked immunosorbent assay (ELISA; $n=3 /$ group). (c) S100A8 expression in colonic epithelial cells (cECs) from A. faecalis monocolonized ( $A$. faecalis), germ-free (GF), or specific-pathogen-free SPF mice was examined by flow cytometry $(n=3-4 / g r o u p)$. Data in b and $\mathbf{c}$ are representatives of two independent experiments and are expressed as mean $\pm \mathrm{s} . \mathrm{d}$.

We previously reported that dextran sodium sulfate-induced colitis (DSS-colitis) is suppressed by the blockade of Dectin-1 signaling. ${ }^{25}$ Thus, we finally examined whether removal of $\beta$ glucans from food could inhibit the development of DSS-colitis. Continuous administration of BGFD from 3 weeks before the treatment with DSS significantly improved the survival rate compared with ND groups (Figure 7c), and the severity score of the inflammatory lesions in BGFD-fed mouse colon was also significantly lower than that in ND-fed mice (Figure 7d,e). Neutrophil infiltration in both cEC and cLP layer was significantly suppressed in BGFD-fed mice (Figure 7f,g). These findings suggest that orally taken $\beta$-glucans are responsible for the activation of Dectin-1, and suppress L. murinus growth by inducing AMPs.

\section{DISCUSSION}

Interaction between commensal bacteria and innate immune receptors plays an important role in maintaining the homeostasis of the intestinal immune system and breakdown of this symbiotic relationship can trigger serious intestinal diseases such as inflammatory bowel disease. ${ }^{10}$ In this study, we identified the detailed mechanisms by which Dectin-1 signaling controls intestinal microbiota. We showed that AMPs, such as S100A8, are indirectly induced in cECs by Dectin-1 signaling through induction of cytokines, especially IL-17F, in CD11 ${ }^{+}$ $\& \mathrm{CD} 11 \mathrm{~b}^{+}$myeloid cells. Furthermore, we showed that Dectin-1 ligands are mainly derived from food components, but not from intestinal microbiota, under our experimental conditions. 


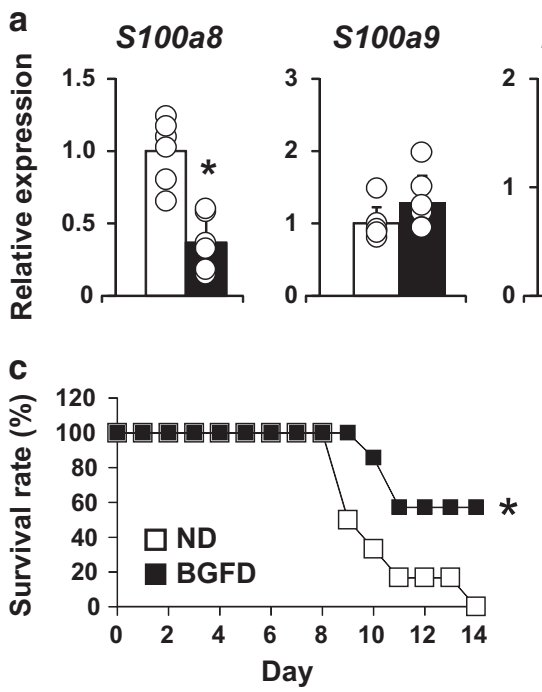

f

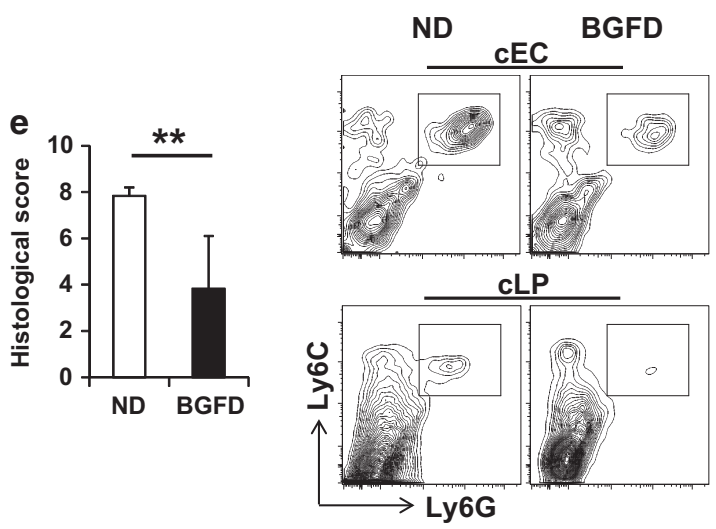

Defb1

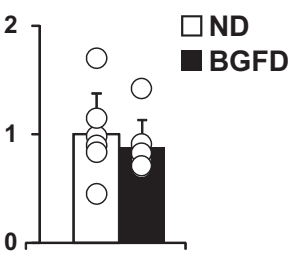

d

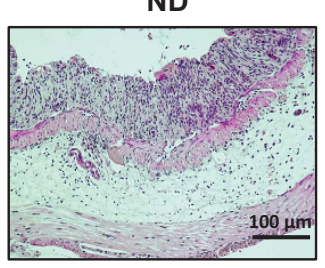

b

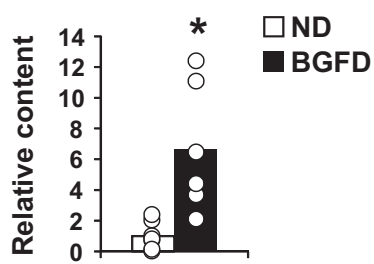

BGFD

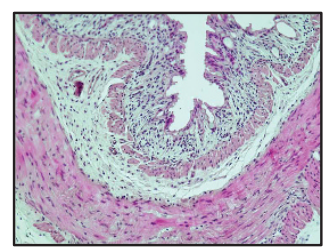

g

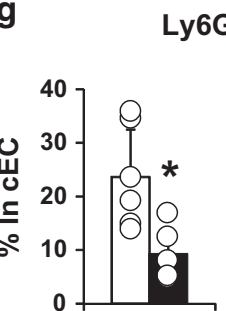

Ly6G+Ly6C+
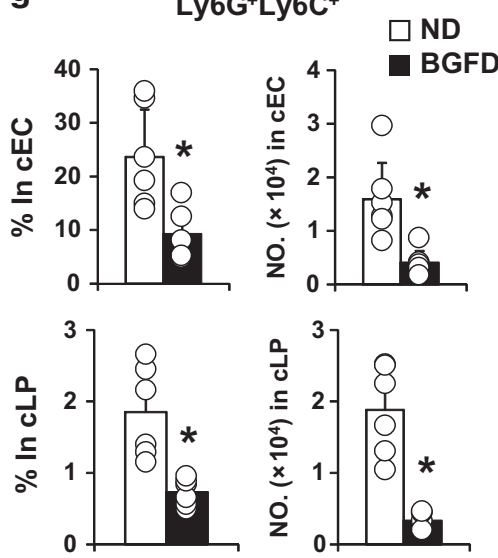

Figure $7 \quad \beta$-Glucans in food are responsible for the activation of colonic Dectin-1. (a,b) Mice were fed with normal diet (ND) or $\beta$-glucan-free diet (BGFD) for 3 weeks, and colonic antimicrobial protein/peptide (AMP) expression (a) and fecal Lactobacillus murinus ribosomal DNA rDNA content (b) were analyzed by quantitative real-time reverse transcription PCR (qRT-PCR; $n=6 /$ group). (c) ND- or BGFD-fed mice were administrated with $2 \%$ dextran sodium sulfate (DSS) in drinking water, and survival rate was evaluated daily ( $n=6-7 /$ group). (d,e) Histological analysis (hematoxylin-eosin (HE) staining) of the colon sections from ND- or BGFD-fed mice was performed on the day 10 after $2 \%$ DSS treatment. (f,g) On day 7 after DSS treatment, mice were killed and neutrophil infiltration in colonic epithelial cell (cEC) or colonic lamina propria (cLP) layer was examined by flow cytometry ( $n=6 /$ group). Data in $\mathbf{a}-\mathbf{g}$ are representatives of two independent experiments and are expressed as mean \pm s.d. ${ }^{\star} P<0.05$, ${ }^{\star \star} P<0.01$ vs. ND control.

Calprotectin is one of the AMPs and also acts as an endogenous TLR4 ligand as well as a chemotactic and immunostimulatory/modulatory factor. ${ }^{30,37}$ In this study, we showed that S100A8-S100A9 preferentially suppresses the growth of L. murinus, but not the growth of E. coli, $L$. lactis, or even L. johnsonii. Interestingly, S100A8 secretion was selectively reduced in $\mathrm{Clec}_{7 a^{-1-}}$ and BGFD-fed mice, accompanied by overexpansion of L. murinus. These observations suggest that Dectin-1 regulates microbiota population in the intestine by regulating AMP secretion. Similar regulation was also reported with other bacteria; intestinal segmented filamentous bacteria, which induces $\mathrm{T}$ helper type 17 cell differentiation and exacerbates autoimmune diseases in their host,${ }^{38}$ is specifically regulated by $\alpha$-defensins that are produced by Paneth cells. ${ }^{39}$ Other PRRs, like NOD2, are also reported to regulate intestinal bacterial microbiota via AMP production in cECs. $^{40}$
Another important finding of this study is the mechanism to induce AMPs downstream of Dectin-1. We showed that Dectin-1 signaling induces IL-17F expression in cLP CD11 $\mathrm{c}^{+}$ $\& \mathrm{CD} 11 \mathrm{~b}^{+}$cells, and this cytokine activates S100A8 expression in cECs. Furthermore, we showed that S100A8 secretion was reduced in $I l 17 f^{-1-}$ mice, resulting in the expansion of $L$. murinus. These results suggest that IL-17F is involved in the induction of S100A8 and the Dectin-1-IL-17F-S100A8lactobacillus axis is important for the intestinal immune homeostasis. However, as IL-17F-deficiency and Dectin-1deficiency did not suppress the expression of S100A8 completely, we think that other PRRs and their downstream cytokines may also participate in this AMP induction. In support for this idea, reduced AMP expression in cECs was reported in cytokine-deficient mice such as IL-17A, IL-22, or IL-23. ${ }^{38,41,42}$ Although IL-23 can induce AMPs in colon organ cultures (Figure 4e,f), IL-23 failed to induce S100A8 
in an intestinal epithelial cell line or primary cECs (Figure $\mathbf{4 g}$ ). These results suggest that S100A8 is indirectly induced by IL-23 through activation of certain cells such as $\mathrm{T}$ cells or myeloid-derived cells in the organ culture other than cECs.

S100A8 and S100A9 are initially identified to be expressed by circulating neutrophils and monocytes as inflammatory factors associated with proinflammatory cell activation and accumulation. ${ }^{43}$ However, because neutrophils are rarely found in the colon under physiological conditions, the main source of S100A8 is considered to be epithelial cells. On the other hand, after induction of colitis, substantial numbers of neutrophils infiltrate into lamina propria and epithelial layer of the colon. Thus, under inflamed conditions, these inflammatory cells may also participate in the production of S100A8.

It is known that both IL-17A and IL-17F are expressed in T helper type 17 cells, $\gamma \delta \mathrm{T}$ cells, and innate lymphoid cell-3. However, in this study, we showed that IL-17F is also produced by myeloid-derived cells in which IL-17A is not produced. The regulatory mechanisms for this preferential expression of IL$17 \mathrm{~F}$ are not known yet. Probably, the chromatin structures surrounding Il17a and Il17f genes are different in CD11c \pm / $\mathrm{CD} 11 \mathrm{~b} \pm$ myeloid-derived cells from lymphoid cells.

Although $A$. faecalis var. myxogenes in soil synthesizes $\beta$ glucans, ${ }^{35,36}$ we showed that $A$. faecalis subsp. Faecalis in the intestine does not produce Dectin-1 ligands. In addition, we could not detect any $\beta$-glucan-expressing microbiota in the intestine, consistent with our recent publication that our specificpathogen-free mouse colony is free from commensal fungi different from the mouse colony of the Underhill group. ${ }^{2}$ Furthermore, the expression levels of S100a8 in cECs were similar between germ-free mice and specific-pathogen-free mice, suggesting that intestinal microflora is not involved in Dectin-1 activation. Instead, decreased S100a8 expression and increased $L$. murinus colonization were observed in mice fed with $\beta$-glucanfree food, suggesting that $\beta$-glucans in foods serve as the main source of Dectin-1 ligand. These findings indicate that $\beta$-glucans in food are an important regulator of intestinal microbiota that regulate intestinal immune system, and may provide us a clue to develop functional foods to prevent inflammatory bowel disease or medicines to treat inflammatory bowel disease.

\section{METHODS}

Mice. Clec7a $a^{-1-}, C_{l e c} 4 n^{-1-}, I l 17 a^{-1-}$, and $I l 17 f^{-1-}$ mice were generated as previously described, ${ }^{14,18,24,44}$ and were used for the experiments after backcrossing for 9 generations to C57BL/6J mice. Control C57BL/6J mice were bred in the same animal room and ageand sex-matched mice were used. $M y d 88^{-/-}$mice were kindly provided by Dr Shizuo Akira (Osaka University, Suita, Japan) and were backcrossed for 9 generations to C57BL/6J mice. All mice were kept under specific pathogen-free conditions with $\gamma$-ray sterilized diet, acidified $(0.002 \mathrm{~N} \mathrm{HCl}, \mathrm{pH} 2.5)$ tap water, and autoclaved wooden chip bed in environmentally controlled clean rooms at the Research Institute for Biomedical Sciences, Tokyo University of Science (Chiba, Japan). Mice were fed with normal diet (F1, Funabashi Farm, Chiba, Japan), except for the experiments in which they were fed with $\beta$ glucan-free food (AIN-93G, Oriental Yeast, Tokyo, Japan) and housed without wooden chip bed. The experiments were carried out according to the institutional ethical guidelines for animal experiments and the safety guidelines for gene manipulation experiments, and were approved by the institutional committees.

Fecal microbiota 16S rDNA analysis. Fresh feces were collected from mice just after the compelled defecation, and digested with $1 \mathrm{mg} \mathrm{ml}^{-1}$ Protease K (Sigma-Aldrich, St Louis, MO) in a buffer containing $50 \mathrm{mM}$ Tris (pH 7.5), $100 \mathrm{mM}$ EDTA, and $0.5 \%$ SDS for $5 \mathrm{~h}$ at $55^{\circ} \mathrm{C}$. Fecal microbiota DNA was then isolated and purified by phenol/ chloroform extraction, followed by ethanol precipitation.

For quantitation of bacteria in feces, total 16S rDNA was amplified from extracted DNA using bacterial universal PCR primers Bact-27F $\left(5^{\prime}\right.$-AGRGTTTGATYMTGGCTCAG-3') and Bact-1492R (5'-GG YTACCTTGTTACGACTT- $\left.3^{\prime}\right)$. The reaction conditions were as follows: $10 \times$ PCR buffer (TaKaRa-Bio, Shiga, Japan), $25 \mathrm{mM}$ dNTP

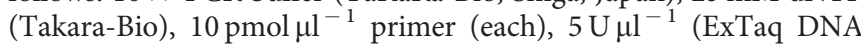
polymerase, Takara-Bio), template DNA, and DNase free water. PCR was performed using a iCycler system (Bio-Rad, Hercules, CA). The following cycling parameters were used: $30 \mathrm{~s}$ of initial denaturation at $96^{\circ} \mathrm{C}, 20 \mathrm{~s}$ annealing at $56^{\circ} \mathrm{C}$, and elongation for $90 \mathrm{~s}$ at $68^{\circ} \mathrm{C}$, with a final extension at $72{ }^{\circ} \mathrm{C}$ for $10 \mathrm{~min}$. Amplified products from all samples were verified by gel electrophoresis using $1 \mu \mathrm{g}$ of the PCR reaction mixture in $1.0 \%$ agarose gels. PCR products were cloned into pCR-4-TOPO vectors (Invitrogen, Carlsbad, CA), and DH12S competent E. coli (Invitrogen) were transformed using the TOPOTA cloning kit for sequencing (Invitrogen). A total of 96 colonies were randomly isolated from each bacterial PCR product. Sequencing templates were prepared by colony PCR using primers M13F ( $5^{\prime}$ GTAAAACGACGGCCAG- $3^{\prime}$ ) and M13R (5'-CAGGAAACAG CTATGAC- $3^{\prime}$ ). PCR products were then treated with exonuclease I and shrimp alkaline phosphatase (GE Healthcare, Waukesha, WI). The $16 \mathrm{~S}$ sequences of the inserts were determined by cycle sequencing using BigDye Terminator (Applied Biosystems, Carlsbad, CA) and 3.2 pmoles of T7 (5'-TAATACGACTCACTATAGGG-3'), T3 (5'AATTAACCCTCACTAAAGGG- $3^{\prime}$ ), and Bact-357F (5'-CCTACG GGAGGCAGCAG- $3^{\prime}$ ) sequencing primers. DNA was cleaned up by ethanol precipitation and sequenced with an automated ABI 3730 capillary sequencers (Applied Biosystems). Each clone data were assembled with the Phred-Phrap program. ${ }^{45}$ The assembled sequences with Phrap were aligned by Clustalw analysis and the multiple-aligned sequences were calculated for the distance matrix of whole sequences that were aligned to the $16 \mathrm{~S}$ rRNA sequences of the RDP (Ribosomal Database Project II) database running in the NCBI (National Center for Biotechnology Information) BLAST (https://rdp.cme.msu.edu).

For the relative quantity of L. murinus, $2 \mathrm{ng}$ of total fecal DNA was used as the template for real-time RT-PCR. The following bacterial specific primers were used: $16 \mathrm{~S}(8 \mathrm{~F} \& \mathrm{R} 357)$ forward $5^{\prime}$-AGAGTTT GATCMTGGCTCAG-3', reverse 5'-CTGCTGCCTYCCGTA-3'; L. murinus (NBRC14221) forward 5'-CGTGGGTAGCAAACA GGATT-3', reverse $5^{\prime}$-TAAGGTTCT TCGCGTTGCTT-3'. Cycle numbers of PCR were used to calculate the relative quantity by normalizing to the amount of total bacterial $16 \mathrm{~S}$ rDNA.

Isolation of cLP cells. Mouse colons were cut out and opened longitudinally, washed with phosphate-buffered saline to remove luminal contents, and cut tissues into small pieces. Tissue pieces were first incubated in a RPMI-1640 (Wako, Osaka, Japan) containing 10\% fetal bovine serum, $1 \%$ penicillin + streptomycin, and $20 \mathrm{mM}$ EDTA for $30 \mathrm{~min}$ at $37^{\circ} \mathrm{C}$ in a shaking water bath, and epithelial cells in the culture supernatant were discarded. After centrifugation (1,500 r.p.m. for $5 \mathrm{~min}$ ), the tissue pellet was further incubated with RPMI containing $10 \%$ fetal bovine serum, $300 \mathrm{U} \mathrm{ml}^{-1}$ collagenase type VIII (Sigma-Aldrich), and $5 \mathrm{U} \mathrm{ml}^{-1}$ DNase I (Sigma-Aldrich) for $2 \mathrm{~h}$ at $37^{\circ} \mathrm{C}$ in a shaking water bath. The digested tissues were then vortexed for 1 min to obtain single-cell suspension. CD11b ${ }^{+}$and CD11c ${ }^{+}$cells from cLP cells were purified to $>90 \%$ by positive selection using a 
mixture of anti-CD11b and anti-CD11c microbeads and auto MACS Pro Separator (Miltenyi Biotec, Bergisch Gladbach, Germany) according to the manufacturer's directions.

Isolation and culture of intestinal epithelial cells. Mouse colons were isolated and cut into $5 \mathrm{~mm} \times 5 \mathrm{~mm}$ pieces and washed with ice-cold phosphate-buffered saline. Tissue pieces were incubated with RPMI1640 containing $10 \%$ fetal bovine serum, $1 \%$ penicillin + streptomycin, and $20 \mathrm{mM}$ EDTA at $37^{\circ} \mathrm{C}$ for $30 \mathrm{~min}$ in a shaking water bath. Then, released epithelial cells were collected, washed for three times by phosphate-buffered saline, and passed through $200 \mu \mathrm{m}$ nylon mesh. Cells were counted, and $5 \times 10^{5} \mathrm{cECs}$ were resuspended with Matrigel (BD Biosciences, San Jose, CA) and then placed into a precold 48-well plate. After solidifying the Matrigel, culture medium was added and the samples were incubated at $37^{\circ} \mathrm{C}$ with $5 \% \mathrm{CO}_{2}$. After $6 \mathrm{~h}$, Dispase (BD Biosciences) was added on the Matrigel medium, and after $1 \mathrm{~h}$, cECs were collected for qRT-PCR analysis.

In vitro culture and measurement of cytokine concentration. Purified cLP-derived CD11 ${ }^{+} \& \mathrm{CD} 11 \mathrm{~b}^{+}$cells $\left(3 \times 10^{5}\right)$ were cultured with curdlan (Sigma-Aldrich) for $6 \mathrm{~h}$ followed by qRT-PCR analysis, or the supernatant of the culture after $72 \mathrm{~h}$ was harvested and cocultured with cECs in Matrigel medium. Cytokine concentration was measured by an enzyme linked immunosorbent assay Development Kit for mouse TNF- $\alpha$ (R\&D Systems, Minneapolis, MN).

Flow cytometry. Antibodies against mouse CD11b (M1/70), CD11c (HL3), CD45 (30-F11), Ly6G (1A8), and Ly6C (HK1.4) were obtained from BioLegend (San Diego, CA), antibodies against IL-17F (18F10) and EpCAM-1 (G8.8) were obtained from eBioscience (San Diego, CA), and antibody against S100A8 (cat. no. BAF3059) was obtained from R\&D Systems. Antibody against mouse S100A9 (2B10) was obtained from BD Biosciences. The 2.4G2 (rat IgG1 anti-FC $\gamma \mathrm{RII} / \mathrm{III}-$ specific mAb-producing hybridoma) was obtained from American Type Culture Collection (Manassas, VA). For intracellular detection of cytokines or AMPs, cells were stimulated with $100 \mu \mathrm{g} \mathrm{ml}^{-1}$ curdlan and $5 \mu \mathrm{g} \mathrm{ml}{ }^{-1}$ Brefeldin A (BD Biosciences) for $3 \mathrm{~h}$ at $37^{\circ} \mathrm{C}$ under $5 \%$ $\mathrm{CO}_{2}$ atmosphere. After cell surface staining, cells were permeabilized with Cytofix/Cytoperm kit (BD Biosciences) and stained with antibodies against intracellular proteins. Detection of $\beta$-glucans on cell surface was carried out using Pi-BGRP. ${ }^{34}$ Cells were first treated with $5 \%$ nonimmunized goat or rabbit serum to inhibit nonspecific binding, and then cells were stained with PE-labeled Pi-BGRP or Pi-BGRP.M, ${ }^{34}$ and the binding was analyzed by fluorescence-activated cell sorting (FACS). Stained cells were analyzed with FACS Canto II and FACS Diva software (BD Biosciences), and Flow Jo software (Tree Star, Ashland, OR) was used for the data analysis. These cells were first gated on FSC-SSC, and gated on $7 \mathrm{AAD}^{-}$to exclude dead cells.

Quantitative real-time reverse transcription PCR (qRT-PCR). Total RNA from colon pieces, cLP cells or cECs was extracted with SepasolRNA I Super G (Nacalai tesque, Kyoto, Japan) according to the manufacturer's instructions. RNA was denatured in the presence of an oligo dT primer and then reverse transcribed with the High Capacity cDNA Reverse Transcription Kit (Applied Biosystems, Foster City, CA). Quantitative PCR was performed with a SYBR Premix Ex Taq (Takara, Kusatsushi, Shiga, Japan) and an iCycler system (Bio-Rad), and the expression of each mRNA encoding cytokine was normalized with Gapdh expression level. Sequences of primer used in this study are described in Supplementary Table S1.

Antibacterial effects of AMPs. L. murinus (NBRC 14221), L. johnsonii (NBRC 13952), Lactococcus lactis (NBRC 100933) and E. coli (NBRC 102203) were cultured in MRS medium (for L. murinus, L. johnsonii, and Lactococcus lactis) and LB medium (for E. coli) respectively, in the presence of recombinant S100A8, S100A8 + S100A9 (1:1 mixture, $5 \mu \mathrm{g} \mathrm{ml}^{-1}$ of each protein, Abcam, Cambridge, UK), or recombinant Lipocarin-2 $\left(5 \mu \mathrm{g} \mathrm{ml}^{-1}, \mathrm{R} \& \mathrm{D}\right)$ for 3 or $9 \mathrm{~h}$ at $37^{\circ} \mathrm{C}$, and bacterial growth was measured with a NanoDrop 2000c spectrophotometer
(Thermo Fisher Scientific, Yokohama, Japan). Both MRS and LB media contain $\mathrm{Mg} 2+$ and other metal ions to avoid the influence of metal ion chelating activity of calprotectin.

Bacterial strain and preparation. L. murinus (NBRC 14221), L. johnsonii (NBRC 13952), E. coli (NBRC 102203), L. lactis (NBRC 100933), and A. faecalis (NBRC 13111) were purchased from National Institute of Technology and Evaluation Biological Resource Center (Kisarazu, Japan). L. murinus, L. johnsonii, and Lactococcus lactis were cultured in Lactobacilli Man-Rogosa-Sharpe broth (Difco, Detroit, MI) at $37^{\circ} \mathrm{C}$ for $18 \mathrm{~h}$ and anaerobic incubation, and E. coli and $A$. faecalis were cultured in lysogeny broth at $37^{\circ} \mathrm{C}$ for $18 \mathrm{~h}$ with aeration before they were used.

DSS-induced colitis. For the induction of acute colitis, mice were administered 2\% (weight/volume) DSS (molecular weight $36-50 \mathrm{kDa}$; MP Biomedicals, Illkirch, France) in their drinking water and survival rate was observed daily. In another experiment, mice were killed on day 7 after DSS administration, and the CLP and cEC cells were prepared for flow cytometry.

Histology. The anus of colitis mouse on day 10 was removed and fixed with $10 \%$ neutral buffered formalin and embedded in paraffin. After cutting in round slices, $5 \mu \mathrm{m}$ thin tissue sections were stained with hematoxylin and eosin. Histological score was calculated using the method of previous research: epithelium (E), $0=$ normal morphology; $1=$ loss of goblet cells; $2=$ loss of goblet cells in large areas; $3=$ loss of crypts; $4=$ loss of crypts in large areas; and infiltration (I), $0=$ no infiltrate; $1=$ infiltrates around the crypt basis; $2=$ infiltrates reaching the lamina muscularis mucosae; $3=$ extensive infiltration reaching the lamina muscularis mucosae and thickening of the mucosa with abundant edema; $4=$ infiltration of the lamina submucosa. ${ }^{46}$ Total histological score was given as $\mathrm{E}+\mathrm{I}$.

Statistical analysis. Differences in parametric data were evaluated by Student's $t$-test or one-way analysis of variance (Bland-Altman) (in Figures $4 \mathbf{c}$ and $\mathbf{5 d})$. Differences in survival rates were evaluated by the log rank test (Mantel-Cox). Histological scores were analyzed using the Mann-Whitney $U$-test. Differences of $P<0.05$ were considered statistically significant.

SUPPLEMENTARY MATERIAL is linked to the online version of the paper at http://www.nature.com/mi

\section{ACKNOWLEDGMENTS}

We thank Sachiko Kubo for her excellent technical assistance. This work was supported by the Science and Technology Research Promotion Program for Agriculture, Forestry, Fisheries and Food Industry (to Y.I.), CREST (Y.I.), and Grants-in Aid from the Ministry of Education, Culture, Sports, Science and Technology of Japan (Y.I. and C.T.).

\section{AUTHOR CONTRIBUTIONS}

T.K. mainly contributed throughout this work and wrote the manuscript. C.T. supervised the experiments and manuscript preparation with help from M.K. T.K., C.T., K.O., and M.H. performed the bacterial 16S rRNA analysis. C.T. and T.K. contributed to the maintenance of germ-free mice. S.S. generated $\mathrm{Clec}_{\mathrm{Ca}}{ }^{-1-}$ mice, and Y.A. and N.O. provided BGRP. Y.I. organized and supervised the project and edited the manuscript.

\section{DISCLOSURE}

The authors declared no conflict of interest.

(c) 2018 Society for Mucosal Immunology

\section{REFERENCES}

1. Round, J. L. \& Mazmanian, S. K. The gut microbiota shapes intestinal immune responses during health and disease. Nat. Rev. Immunol. 9, 31323 (2009). 
2. Iliev, I. D. et al. Interactions between commensal fungi and the c-type lectin receptor Dectin-1 influence colitis. Science 336, 13141317 (2012).

3. O'Hara, A. M. \& Shanahan, F. The gut flora as a forgotten organ. EMBO Rep. 7, 688-93 (2006).

4. Cash, H. L., Whitham, C. V, Behrendt, C. L. \& Hooper, L. V. Symbiotic bacteria direct expression of an intestinal bactericidal lectin. Science 313, 1126-30 (2006).

5. Hooper, L. V., Stappenbeck, T. S., Hong, C. V \& Gordon, J. I. Angiogenins: a new class of microbicidal proteins involved in innate immunity. Nat. Immunol. 4, 269-73 (2003).

6. Ivanov, I. I. \& Honda, K. Intestinal commensal microbes as immune modulators. Cell Host Microbe 12, 496-508 (2012).

7. Kosiewicz, M. M., Zirnheld, A. L. \& Alard, P. Gut microbiota, immunity, and disease: a complex relationship. Front. Microbiol. 2, 180 (2011).

8. Guarner, F. Enteric flora in health and disease. Digestion 73, 5-12 (2006).

9. Rakoff-Nahoum, S., Paglino, J., Eslami-Varzaneh, F., Edberg, S. \& Medzhitov, R. Recognition of commensal microflora by toll-like receptors is required for intestinal homeostasis. Cell 118, 229-41 (2004).

10. Maloy, K. J. \& Powrie, F. Intestinal homeostasis and its breakdown in inflammatory bowel disease. Nature 474, 298-306 (2011).

11. Figuredor, C. G., Kooyk, Y., Van, \& Adema, G. J. C-type lectin receptors on decdritic cells and langerhans cells. Nat. Rev. Immunol. 2, 77-84 (2002).

12. Marakalala, M. J., Graham, L. M. \& Brown, G. D. The role of Syk/CARD9coupled C-type lectin receptors in immunity to Mycobacterium tuberculosis infections. Clin. Dev. Immunol. 2010, 567571 (2010).

13. Saijo, S. \& Iwakura, Y. Dectin-1 and Dectin-2 in innate immunity against fungi. Int. Immunol. 23, 467-72 (2011).

14. Saijo, S. et al. Dectin-2 recognition of alpha-mannans and induction of Th17 cell differentiation is essential for host defense against Candida albicans. Immunity 32, 681-691 (2010).

15. Yonekawa, A. et al. Dectin-2 is a direct receptor for mannose-capped lipoarabinomannan of mycobacteria. Immunity 41, 402-413 (2014).

16. Brown, G. D. et al. Dectin-1 is a major beta-glucan receptor on macrophages. J. Exp. Med. 196, 407-412 (2002).

17. Taylor, P. R. et al. Dectin-1 is required for $\beta$-Glucan recognition and control of fungal infection. Nat. Immunol. 8, 31-8 (2007).

18. Saijo, S. et al. Dectin-1 is required for host defense against Pneumocystis carinii but not against Candida albicans. Nat. Immunol. 8, 39-46 (2007).

19. Hiemstra, P. S. Epithelial antimicrobial peptides and proteins: their role in host defence and inflammation. Paediatr. Respir. Rev. 2, 306-310 (2001).

20. Kolls, J. K., McCray, P. B. \& Chan, Y. R. Cytokine-mediated regulation of antimicrobial proteins. Nat. Rev. Immunol. 8, 829-835 (2008).

21. Ganz, T. Defensins: antimicrobial peptides of innate immunity. Nat. Rev. Immunol. 3, 710-20 (2003).

22. Lai, Y. \& Gallo, R. L. AMPed up immunity: how antimicrobial peptides have multiple roles in immune defense. Trends Immunol. 30, 131-141 (2009).

23. Cua, D. J. \& Tato, C. M. Innate IL-17-producing cells: the sentinels of the immune system. Nat. Rev. Immunol. 10, 479-489 (2010).

24. Ishigame, H. et al. Differential roles of interleukin-17A and -17F in host defense against mucoepithelial bacterial infection and allergic responses. Immunity 30, 108-119 (2008).

25. Tang, C. et al. Inhibition of Dectin-1 signaling ameliorates colitis by inducing lactobacillus-mediated regulatory T cell expansion in the intestine. Cell Host Microbe 18, 183-197 (2015).
26. Viemann, D. et al. Myeloid-related proteins 8 and 14 induce a specific inflammatory response in human microvascular endothelial cells. Blood 105, 2955-2962 (2005).

27. Pilling, D., Fan, T., Huang, D., Kaul, B. \& Gomer, R. H. Identification of markers that distinguish monocyte-derived fibrocytes from monocytes, macrophages, and fibroblasts. PLOS ONE 4, e7475 (2009).

28. Zindl, C. L. et al. IL-22-producing neutrophils contribute to antimicrobial defense and restitution of colonic epithelial integrity during colitis. Proc. Natl. Acad. Sci. USA 110, 12768-73 (2013).

29. Corbin, B. D. et al. Metal chelation and inhibition of bacterial growth in tissue abscesses. Science 319, 962-965 (2008).

30. Vogl, T. et al. Mrp8 and Mrp14 are endogenous activators of Toll-like receptor 4, promoting lethal, endotoxin-induced shock. Nat. Med. 13, 1042-1049 (2007).

31. Xu, K., Geczy, C. L. \& Sa, C. S. P. IFN- $\gamma$ and TNF regulate macrophage expression of the chemotactic S100 protein S100A8. J. Immunol. 164, 4916-4923 (2000).

32. Song, $X$. et al. IL-17RE is the functional receptor for IL-17C and mediates mucosal immunity to infection with intestinal pathogens. Nat. Immunol. 12, 1151-8 (2011).

33. Reid, D. M., Gow, N.A. \& Brown, G. D. Pattern recognition: recent insights from Dectin-1. Curr. Opin. Immunol. 21, 30-7 (2009).

34. Kanagawa, M., Satoh, T., Ikeda, A., Adachi, Y., Ohno, N. \& Yamaguchi, Y. Structural insights into recognition of triple-helical beta-glucans by an insect fungal receptor. J. Biol. Chem. 286, 29158-65 (2011).

35. Saitô, H., Ohki, T. \& Sasaki, T. A 13C nuclear magnetic resonance study of gel-forming (1 goes to 3)-beta-d-glucans. Evidence of the presence of single-helical conformation in a resilient gel of a curdlan-type polysaccharide 13140 from Alcaligenes faecalis var. myxogenes IFO 13140. Biochemistry 16, 908-14 (1977).

36. Phillips, K. R. et al. Production of curdlan-type polysaccharide by Alcaligenes faecalis in batch and continuous culture. Can. J. Microbiol. 29, 1331-8 (1983).

37. Ehrchen, J. M., Sunderkötter, C., Foell, D., Vogl, T. \& Roth, J. The endogenous Toll-like receptor 4 agonist S100A8/S100A9 (calprotectin) as innate amplifier of infection, autoimmunity, and cancer. J. Leukoc. Biol. 86, 557-66 (2009).

38. Wu, H.-J. et al. Gut-residing segmented filamentous bacteria drive autoimmune arthritis via T helper 17 cells. Immunity 32, 815-27 (2010).

39. Salzman, N. H. et al. Enteric defensins are essential regulators of intestinal microbial ecology. Nat. Immunol. 11, 76-83 (2010).

40. Petnicki-ocwieja, T., Hrncir, T., Liu, Y., Biswas, A. \& Hudcovic, T. Nod2 is required for the regulation of commensal microbiota in the intestine. Proc. Natl. Acad. Sci. USA 106, 15813-15818 (2009).

41. Gessner, M.Aet al. Dectin-1-dependent interleukin-22 contributes to early innate lung defense against Aspergillus fumigatus. Infect. Immun. 80, 410417 (2012).

42. Cayatte, C. et al. Biomarkers of therapeutic response in the IL-23 pathway in inflammatory bowel disease. Clin. Transl. Gastroenterol. 3, e10 (2012).

43. Hessian, P.A., Edgeworth, J \& Hogg, N MRP-8 and MRP-14, two abundant $\mathrm{Ca}(2+)$-binding proteins of neutrophils and monocytes. J. Leukoc. Biol. 53, 197-204 (1993).

44. Nakae, S. et al. Antigen-specific T cell sensitization is impaired in I-17deficient mice, causing suppression of allergic cellular and humoral responses. Immunity 17, 375-387 (2002).

45. Gordon, D. et al. Automated finishing with Autofinish. Genome Res. 11, 614 625 (2001).

46. Obermeier, F. et al. CpG motifs of bacterial DNA exacerbate colitis of dextran sulfate sodium-treated mice. Eur. J. Immunol. 32, 2084-2092 (2002). 